\title{
Demand side management based charging strategy for fleet of plug-in hybrid electric vehicles
}

\author{
Sachpreet Kaur $^{1, ~ *}$, Ravtej Singh Sandhu ${ }^{2}$, Tarlochan Kaur ${ }^{1}$, and Rintu Khanna ${ }^{1}$ \\ ${ }^{1}$ Punjab Engineering College, Electrical Department, Chandigarh, India \\ ${ }^{2}$ Symbiosis Institute of Business Management, Pune, India
}

\begin{abstract}
In coming years, the widespread use of Plug-in Hybrid Electric Vehicles (PHEVs) will impose a significant burden on the existing electric grid. The situation may worsen due to uncontrolled charging strategies adopted for PHEVs. On the other hand, these PHEVs, if charged through proper control mechanisms may reduce additional dynamic load demands. Also, if utilized properly, they may provide significant support to electric grid from time to time. The entire process of regulating the power exchanged with PHEVs w.r.t the existing grid conditions is well known as Demand Side Management (DSM). To indulge PHEVs in DSM, an accurate estimate of characteristics of PHEVs, both on-road and off-road, is necessary. Thus, this study aims to mathematically model the behaviour of four imperative parameters of PHEVs. These are dynamic travel behaviour, battery state-of-charge (SOC) requirements, the energy demands of PHEVs and, total power exchanged by PHEVs with the electric grid. In addition to this, a smart charging strategy is proposed and tested to verify the ability of PHEVs for participating in DSM for peak load management. The impacts of uncontrolled charging and smart charging of PHEVs on grid power demands are also discussed.
\end{abstract}

\section{Introduction}

The random charging of Plug-in Hybrid Electric Vehicles (PHEVs) may impose severe load demands on distribution networks, thus causing various harmful effects. The major effects include transformer overloading, frequency deviations, and voltage fluctuations [1]. On the other hand, charging PHEVs through smart control techniques allows them to exploit their potential to provide grid ancillary services. Also, the controlled charging reduces the burden on the electric grid significantly. The process of regulating load demands on the distribution side is known as Demand-Side Management (DSM) [2]. DSM techniques inhibit different behaviors during contingency and non-contingency events. The main motive for DSM is to utilize existing grid infrastructure efficiently for handling increased electricity load demand, without the need to install additional generation units. So far, the techniques suggested for DSM majorly aim at proposing incentive-based actions to control electricity usage effectively. Some widely adopted economic incentives include real-time pricing, peak time rebate, and critical peak pricing [3].

The increasing penetration of PHEVs in electric grids day by day, demands a particular interest in integrating DSM techniques with charging infrastructures. A statistical analysis conducted in [4] revealed that the probability of PHEVs staying at home is 0.5 and for staying at home during mid-day is 0.9. Thus, there is significant scope for participating PHEVs in DSM. The usage of a PHEV for DSM is highly dependent upon its user's convenience and travel schedule. In addition, to indulge PHEVs in DSM, proper control strategies incorporated with correct information regarding PHEV's travel behavior, battery rating, energy demand, and initial state-of-charge are required [4]. Moreover, since the management of a fleet of PHEVs is a highly complex process, an accurate estimate of the availability of PHEVs off-road for charging is required at all instants of time. Previously, some studies have been conducted to estimate the effect of PHEVs on the existing electric grid [5]. The authors in [5] observed that wide-scale adoption of EVs will have a significant impact on power generation capacities and residential distribution systems. The study conducted in [6], revealed the harmonic distortion effects of PHEV charging loads on the distribution system. Authors in [7], discussed in detail the technical specifications involved for the adoption of PHEVs. In recent years, the literature is witnessing a continuous drift towards the decentralized approach for PHEV charging. In this approach, PHEVs owners have complete involvement in charging decisions [8]. The authors in [9] coordinated the aggregate of PHEVs for participation in frequency regulation. They investigated the role of PHEVs in fulfilling grid power requirements using a suitable Fuzzy Logic Controller (FLC). The authors in [10] proposed an adaptive controller for managing power requirements of PHEVs for load management in microgrids. However, the authors lack in discussing the role of incentives in smart charging. Several price-based charging frameworks have been proposed in the literature for volunteer participation of PHEV users in DSM [11]. In comparison to existing flat-rate pricing incentive, the real-time pricing incentives has been

* Corresponding author: sachpreetkaur.phdele16@pec.edu.in 
considered as the finest cost-effective alternative for both PHEV users and utility. It prevents overcharging of PHEVs during peak hours [12]. Another useful work reported in [13] discussed the optimized charging of PHEVs, in order to reduce their usage cost. In [14], the authors proposed an energy cost-sharing model based on game theory to encourage PHEV owners to participate in coordinated charging and discharging.

Summarizing the above, it has been observed that the major portion of literature focuses on the study of harmful effects of integrating PHEVs with existing electric grids. Some researchers emphasized the need for DSM using PHEVs. However, in order to achieve optimal scheduling of PHEV s and to effectively indulge them in DSM, the government organizations and utilities must be able to forecast total charging load demands of PHEVs, at all instants of time. Also, since the adoption of PHEVs on a wide scale is a futuristic approach, accurate modelling of PHEV load demand is desired. Keeping in concern the above, this study aims to model mathematically the four imperative parameters of PHEVs. These include the travel behaviour of PHEVs, energy demands of PHEVs, State-of-Charge (SOC), and total power exchanged between the PHEV and electric grid. Initially, statistical tools have been designed to predict the load demands of PHEVs during different times of the day. Thereafter, a smart charging strategy has been proposed and tested to analyse the ability of PHEVs to participate in DSM. The remainder of the paper is organized in the following manner. Section 2 discusses the mathematical modelling of load demand of PHEVs, Section 3 explains two different charging scenarios of PHEVs, Section 4 compiles the results along with the discussions and Section 5 considers the conclusions observed during analysis.

\section{Mathematical Modelling of Load Demand of PHEVs}

The adoption of PHEVs as dynamic storage buffers in balancing load demands and power generation is gaining attention worldwide. In recent years, Nickel Metal Hydride $(\mathrm{NiMH})$, lead-acid and lithium-ion have been the most widely used batteries in PHEVs. This is due to various reasons including their performance characteristics and economic profits [15]. In this study, PHEVs equipped with lithium-ion batteries are considered for analysis purposes.

In order to determine load demands by PHEVs, statistical behaviour and analysis is essential. Since the behaviour of PHEVs on-road and off-road is highly dynamic in nature, the present study assumes the plug-in time of a PHEV to be a random variable, with Probability Density Function (pdf), $f(t)$. The two important parameters: the initial SOC of battery $\left(S O C_{i}\right)$ and the electricity tariff structure highly influence the randomness of plug-in time of PHEVs. Since the $S O C_{i}$ of a PHEV's battery is dependent upon the travel behaviour of PHEV's users and the total distance they travel in a day, the present study also assumes the $S O C_{i}$ to be a random variable varying between the range $0-100 \%$ [16]. As a result, in order to analyse $S O C_{i}$, the correct estimate of distance travelled by $\mathrm{PHEV}$ is required. Based on the information available in [17], a pdf of daily distance travelled in a day is obtained. As seen in the curve shown in Figure 1, it is observed that the distribution pattern is of 'lognormal type', where the probability of occurrence of negative distance is zero, and that of positive distance extends till infinity. Eq. (1) computes the pdf for the travel behaviour of PHEV w.r.t the distance travelled.

$$
p(d ; \mu, \sigma)=\frac{1}{d \sqrt{2 \pi \sigma^{2}}} e^{\frac{-(\ln d-\mu)^{2}}{2 \sigma^{2}}}, d>0
$$

In Eq. (1) ' $d$ ' represent the distance travelled by PHEV, $\mu$ is the mean, and $\sigma$ is the standard deviation. Based on data extracted from [17], the mean and standard deviation of distance travelled are 17.89 miles and 9.86 miles respectively.

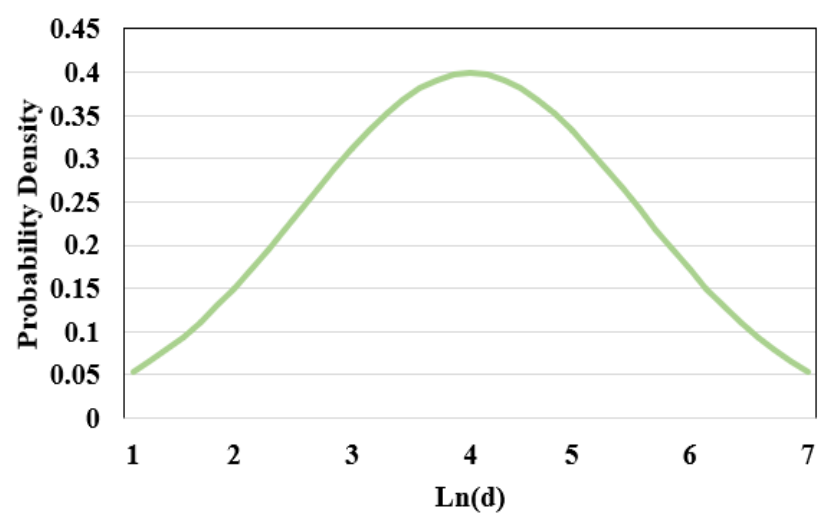

Fig.1. Probability Density Function of daily distance travelled by PHEV users

Given the statistical analysis of distance travelled ' $d$ ', the $S O C_{i}$ of PHEV can be estimated using Eq. (2) as [16],

$$
S O C_{i}=\left(1-\frac{\alpha d}{d_{m}}\right) \times 100 \%
$$

In the above-mentioned equation, ' $d_{m}$ ' represents the maximum range of $\mathrm{EV}$ and ' $\alpha$ ' is the number of days the EV has travelled since the last plug-in (here $\alpha$ is assumed as 1). The pdf curve of $S O C_{i}$ depicted in Figure 2 is expressed in Eq. (3) and is formulated using Eqs. (1) and (2) as,

$$
\begin{aligned}
& s(S O C ; \mu, \sigma)= \frac{1}{\frac{d_{m}}{\alpha}(1-S O C) \sqrt{2 \pi \sigma^{2}}} \\
& \times e-\frac{\left|\ln (1-S O C)-\left(\mu-\ln \left(\frac{d_{m}}{\alpha}\right)\right)\right|^{2}}{2 \sigma^{2}} \\
&, 0<S O C<1
\end{aligned}
$$

Depending upon 'SOC $C_{i}$, the energy and power demands of a PHEV can be computed using Eq. (4) and Eq. (5) respectively.

$$
\begin{aligned}
E_{i} & =\left(1-S O C_{i}\right) E_{r} \\
\int_{t p_{\text {in }}}^{t p_{\text {out }}} P_{i}(t) d t & =\left(1-S O C_{i}\right) E_{r}
\end{aligned}
$$

In the above-mentioned equations, ' $t p_{\text {in }}$ ' and ' $t p_{\text {out }}$ ' represent PHEV's plug-in and plug-out times 
respectively. $E_{i}$ represents the energy requirements of PHEV and ' $P_{i}(t)$ ' represents the power demanded by PHEV.

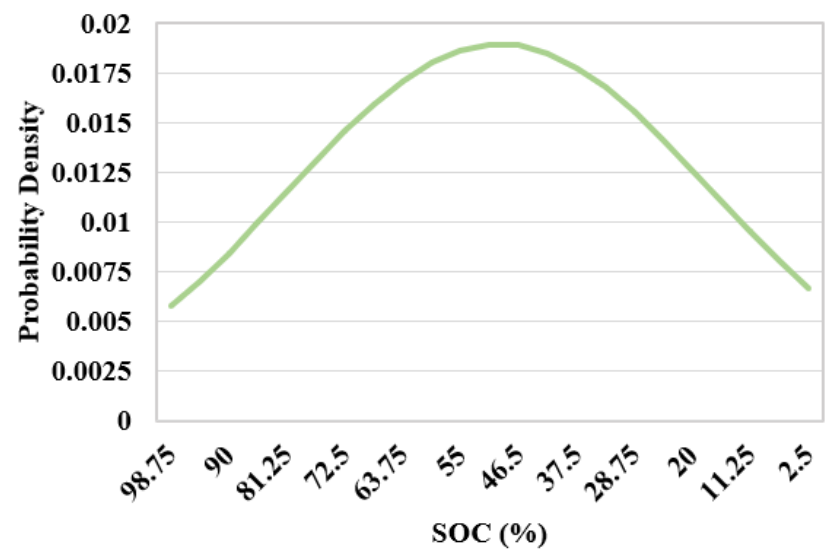

Fig.2. Probability Density Function of SOC of plugged-in PHEV

The power demand of a PHEV varies throughout the charging process according to its battery characteristics as observed in [18]. Also, the power demand occurs independently and varies irrespective of $S O C_{i}$. To conduct numerical calculations, the total power demanded by PHEV battery has been divided into $\left(t p_{\text {out }}-t p_{\text {in }}\right) / 2$ intervals where $\left(t p_{\text {out }}-t p_{\text {in }}\right)$ represents the time required for the entire charging process. The power level $P_{c}$ is then expressed as Eq. (6),

$$
P_{c}=\frac{P((c-1) \Delta t)+P(c \Delta t)}{2}
$$

Where, $\Delta t$ is the length of each interval (here $\Delta t=0.5$ and $\left.t p_{\text {out }}-t p_{\text {in }}=5\right)$. The probability of PHEV charging load, operating at power $P_{c}$ at time $\mathrm{t}$ is represented as $\varnothing\left(P_{c}, t\right)$. The total charging load at any load for a single PHEV is obtained using Eq. (7),

$$
\mu(P)=\sum_{c=1}^{\left(t p_{\text {out }}-t p_{\text {in }}\right) / 2} P_{c} \varnothing\left(P_{C}, t\right)
$$

For multiple batteries, the overall charging demand in a system is computed as the summation of power levels of ' $n$ ' number of PHEVs and is expressed as Eq. (8),

$$
P_{n}=\sum_{i=1}^{n} \sum_{c=1}^{\left(t p_{\text {out }}-t p_{\text {in }}\right) / 2} P_{c} \emptyset\left(P_{c}, t\right)
$$

\section{Charging scenarios for PHEVs}

In this study, two different charging scenarios i.e. Uncontrolled charging and Controlled charging are proposed. During uncontrolled charging, the PHEVs randomly plug-in to charge according to their convenience. In Controlled charging, every PHEV charges through a proper control mechanism, thus participating in DSM. To implement the two control scenarios, three tariff structures are considered. These are flat-rate tariff, time-of-use tariff, and real-time tariff. In a flat-rate tariff-based approach, energy cost $/ \mathrm{kWh}$ remains constant throughout the day. In the time of use based approach, off-peak times, and peak times have different energy cost/kWh. Real-time tariff considers an adaptive approach that constantly monitors the tariff structure in a day depending upon the contingency and non-contingency events. Figure 3. depicts the normalized electricity tariff structure [12].

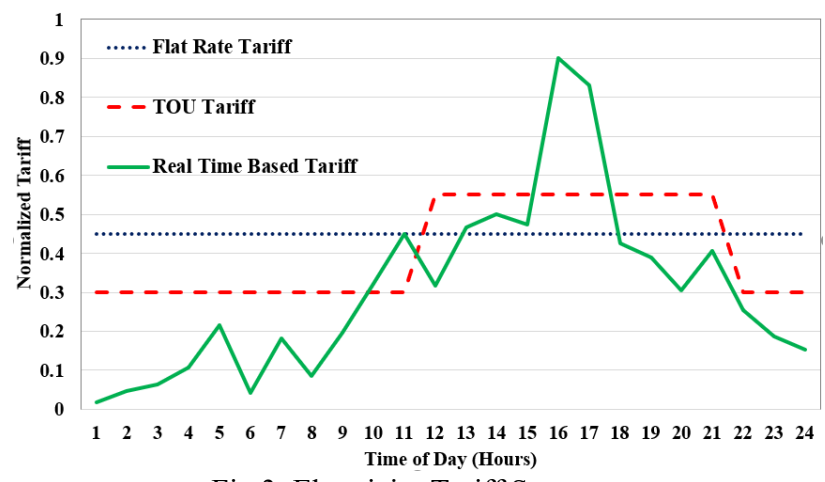

Fig.3. Electricity Tariff Structure

\subsection{Uncontrolled Charging}

In this scenario, a flat-rate tariff-based charging strategy is adopted i.e. government authorities do not provide any incentives to PHEV users for charging. This simply means that the PHEV users start charging anytime according to their convenience and requirement. To analyze the worstcase scenario, it is assumed that all PHEV's begin to charge simultaneously at 17:00. As a result, a uniform probability density function $p d f f_{l}(t)$ of PHEV charging start time is adopted, as described in Eq. (9),

$$
\begin{gathered}
f_{1}(x)=\left\{\begin{aligned}
1.00, & t=17 \\
0, & t=\text { some other time }
\end{aligned}\right. \\
,(1 \leq t \leq 24)
\end{gathered}
$$

The total amount of load imposed by PHEVs at time ' $t$ ' can be computed using the above equation by incorporating an appropriate battery profile and $S(S O C ; \mu, \sigma)$.

\subsection{Controlled Charging}

In this scenario, time-of-use based or real-time tariff based PHEV charging strategy is adopted, to determine the most economic time for plugging-in PHEVs for charging. For this purpose, two main parameters i.e. real-time traffic data and real-time tariff data are required. Figure 4 shows the distribution of PHEV's initial charging time obtained by plotting the reciprocal of the product of two above mentioned parameters. It is observed that the resultant distribution of plug-in time is nearly bell-shaped with the mean around (24:00). Thus, a Gaussian distribution function has been employed to compute ' $p d f$ ' of battery plug-in time as expressed in Eq. (10),

$$
f(d ; \mu, \sigma)=\frac{1}{\sqrt{2 \pi \sigma^{2}}} e^{\frac{-(\mathrm{x}-\mu)^{2}}{2 \sigma^{2}}}
$$

The main focus of DSM based smart charging of PHEVs is to determine optimal charging schedules, to minimize overall economic costs required to satisfy PHEV's owners. Also, it requires effective management of available energy resources with additional monetary benefits provided to 
PHEV users. The mathematical modelling of PHEV smart charging is represented in Eq. (11),

$$
\min \sum_{k=1}^{n} C_{k}(t) P_{k}(t)
$$

Where the number of PHEVs vary from $\mathrm{k}=1 \ldots \mathrm{n}, C_{k}(t)$ is the real-time cost function and $P_{k}(t)$ is the PHEV load at a particular instant of time. The cost function $C_{k}(t)$ at any time $t$ is expressed in Eq. (12).

$$
C_{k}(t)=C_{\text {grid }}(t)+C_{\text {battd }}(t)
$$

In the above-mentioned equations, $C_{\text {grid }}(t)$ refers to the grid operating cost and $C_{\text {battd }}(t)$ refers to the battery degradation cost. While allowing PHEVs for smart charging, certain uncertainties need to be considered. The charging pattern should strictly observe PHEV battery rating, charger specifications, bus voltage, and power flow constraints.

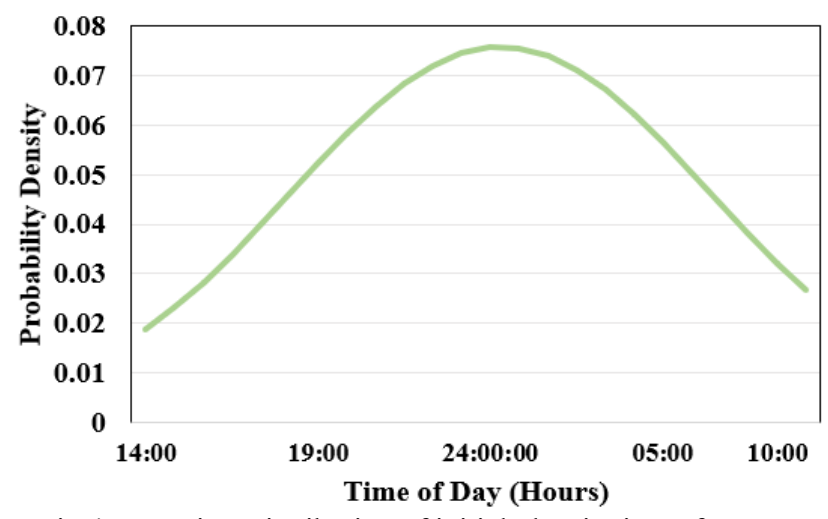

Fig.4. Gaussian Distribution of initial plug-in time of PHEVs

\section{Results and Discussion}

In this study, a small-scale solar-powered microgrid has been studied to determine the impact of PHEV's charging load on the system. The proposed microgrid consists of a photovoltaic power plant, diesel generation unit, and PHEV charging infrastructure The microgrid is supposed to feed residential sector load demands. The load demand data for the residential sector has been obtained from [19] and is plotted in Figure 5. It is observed from the obtained curve, that the load demand reaches it's peak in between $\mathrm{t}=17: 00-19: 00 \mathrm{~h}$. The load curve observes off-peak behavior at $\mathrm{t}=5: 00 \mathrm{~h}$. The smart scheduled charging mechanism strictly monitors the peak load and off-peak load contingency events, to regulate and schedule PHEV charging. The minimization of objective function expressed in Eq. (11), acts as the base for the scheduling process during controlled charging. The PHEV charging infrastructure can integrate a total of 200 PHEVs simultaneously. However, in this study three different penetration levels of PHEVs are considered : $0 \%$ i.e. no PHEV present, $25 \%$ equivalent to $50 \mathrm{EVs}$ and $50 \%$ equivalent to $100 \mathrm{EVs}$. In order to analyze load demands, charging characteristics of a $16.5 \mathrm{kWh}$ lithium-ion battery has been considered [16].

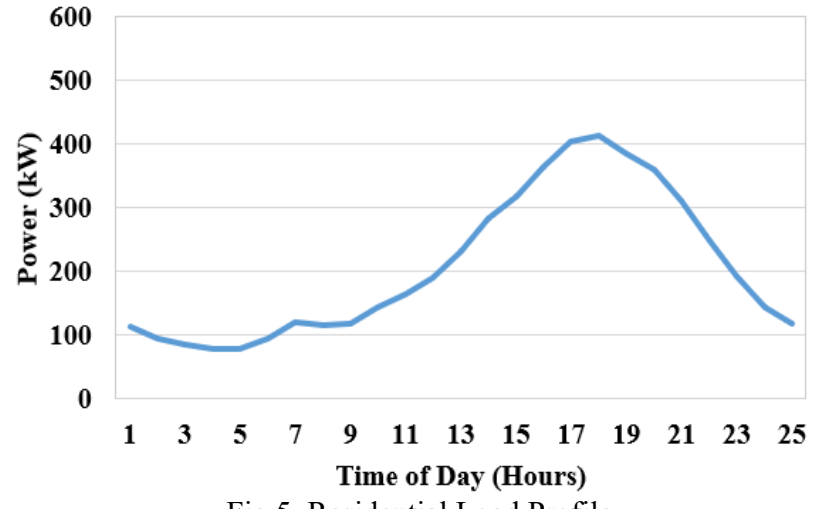

Fig.5. Residential Load Profile

The $S O C_{i}$ and $d$ charactersitics for $12.5 \%$ penetration of PHEVs have been shown in Figure 6. It is clearly observed that more the distance travelled, lesser is the SOC availability in PHEVs for grid support.

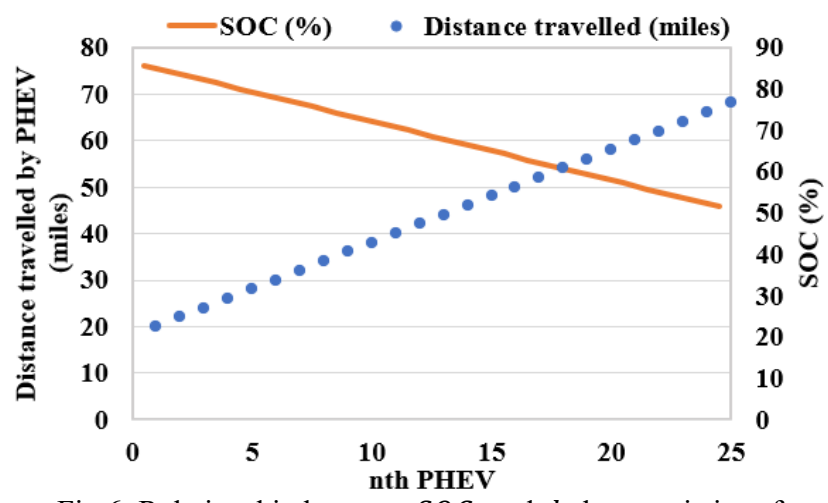

Fig.6. Relationship between $S O C_{i}$ and $d$ characteristics of $\mathrm{PHEV}_{i}$

The load modelling of PHEV is done in two different scenarios: Scenario 1-Uncontrolled charging and Scenario 2-Controlled charging. Figure 7 and Figure 8 depict the load behavior of PHEV in Scenario 1 and Scenario 2 respectively. As observed in Figure 7, during uncontrolled charging, a significant additional peak load originates due to the PHEV charging load at 17:00. The impact analysis of PHEV on peak load is done for different penetration levels of vehicles. The maximum peak originates for $50 \%$ penetration of PHEV. It is clearly observed that even a portion of PHEVs i.e. $25 \%$ penetration in the electric grid, causes a significant impact on load patterns. The peak may further deteriorate with increase in number of PHEVs. The imbalance originated in load-generation may cause various effects in the grid including voltage fluctuations and frequency deviations.

On the other hand, the results obtained after applying DSM based smart charging are depicted in Figure 8. In this scenario, the PHEV power load during different instants of time is based on the optimization of objective function proposed in Eq. (11). The results obtained indicate that the PHEV charging does not generates high peak loads as seen in Scenario 1. Figure 8 reveals the results obtained during the smart charging of PHEVs. The obtained curves show the efficiency of the proposed smart strategy in coordinating EV charging. It is observed that rather than showing a single peak rise in 
load demand, the load demand in Scenario 2 is distributed due to PHEV scheduled charging.

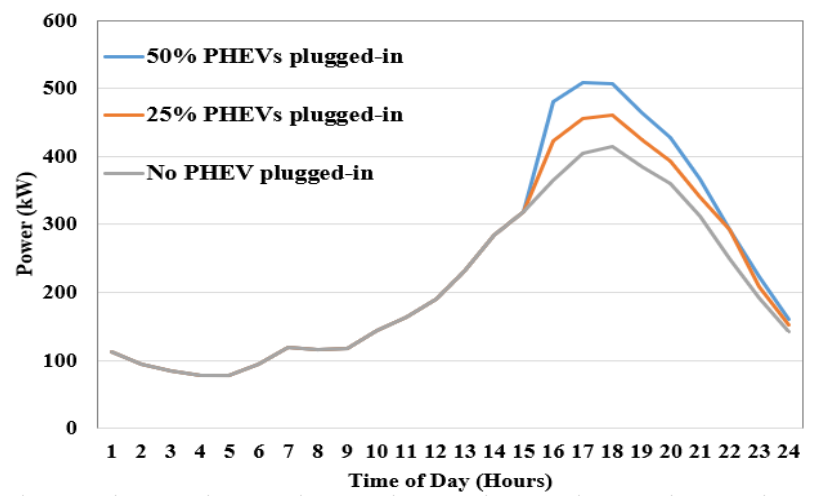

Fig.7. Uncontrolled PHEV charging

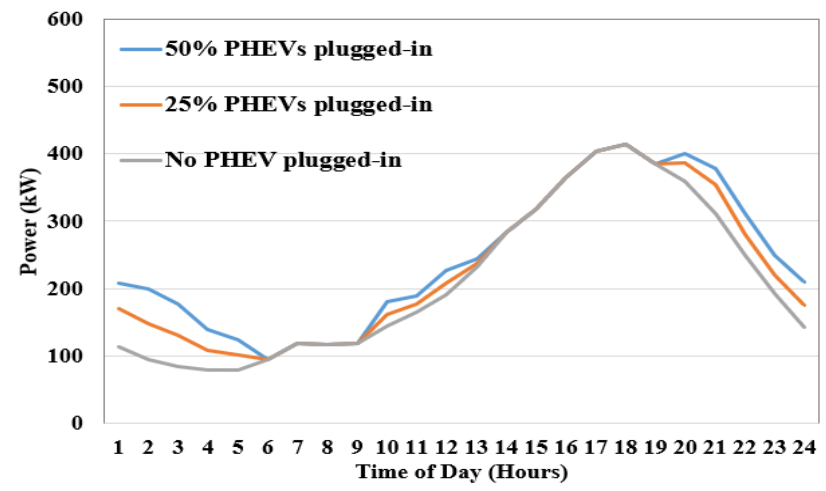

Fig. 8. Controlled PHEV charging

\section{Conclusions and Future Scope}

The present paper employs mathematical modelling to analyze the ability of PHEVs to participate in demand-side management. Statistical survey of behaviour of PHEVs for providing grid regulation is obtained. Further, two different charging strategies, uncontrolled charging and controlled charging, are proposed and discussed. It is observed that during uncontrolled charging, a significant peak load is observed in the distribution system. On the other hand, the proposed coordinated PHEV charging strategy helps to reduce the PHEV load burden on electric grids.

As an extension to this analysis, the influence of PHEVs on important parameters of grid like voltage and frequency could be analyzed. The obtained characteristics will be helpful to develop a suitable control algorithm for PHEVs to provide grid support.

\section{References}

[1] S. Soheil, S. Filizadeh, and E. Bibeau, "Profile of charging load on the grid due to plug-in vehicles," IEEE Trans. Smart Grid, 3, 135-141 (2012)

[2] J. Zazo, S. Zazo, and S. V. Macua, "Robust worst case analysis of demand-side management in smart grids," IEEE Trans. Smart Grid, 8, 662-673 (2017)

[3] Mou, Yuting, Hao Xing, Zhiyun Lin, and Minyue Fu. "Decentralized optimal demand-side management for
PHEV charging in a smart grid." IEEE Transactions on Smart Grid, 6, 726-736 (2014)

[4] Quirós-Tortós, Jairo, Luis F. Ochoa, and Becky Lees. "A statistical analysis of EV charging behavior in the UK." In 2015 IEEE PES Innovative Smart Grid Technologies Latin America (ISGT LATAM), 445-449 (2015)

[5] J. T. Salihi, "Energy requirements for electric cars and their impact on electric generation and distribution systems," IEEE Trans. Ind. Appl.5, 516-531 (1973)

[6] J. C. Gomez and M. M. Morcos, "Impact of EV battery chargers on the power quality of distribution systems," IEEE Trans. Power Del.,18, 975-981, (2003)

[7] Kaur, Sachpreet, Tarlochan Kaur, Rintu Khanna, and Parampal Singh. "A state of the art of DC microgrids for electric vehicle charging." In 2017 4th International Conference on Signal Processing, Computing and Control (ISPCC), 381-386 (2017).

[8] M. C. Falvo, G. Graditi, and P. Siano, "Electric vehicles integration in demand response programs," in Proc. Int. Symp. Power Electron. Elect. Drives Autom. Motion, 548553 (2014)

[9] Falahati, Saber, Seyed Abbas Taher, and Mohammad Shahidehpour. "Grid frequency control with electric vehicles by using of an optimized fuzzy controller." Applied energy, 178, 918-928 (2016).

[10] Kaur, Sachpreet, Tarlochan Kaur, and Rintu Khanna. "ANFIS Based Frequency Control in an Autonomous Microgrid Integrated with PV and Battery Storage." In 2019 9th International Conference on Power and Energy Systems (ICPES), 1-4 (2019)

[11] Schuller, Alexander. "Charging coordination paradigms of electric vehicles." In Plug in Electric Vehicles in Smart Grids, Springer, Singapore, 1-21 (2015). [12] L. Jia and L. Tong, "Dynamic pricing and distributed energy management for demand response," IEEE Trans. Smart Grid, 7, 1128-1136 (2016).

[13] S. Sarabi and L. Kefsi, "Electric vehicle charging strategy based on a dynamic programming algorithm," in Proc. Int. Conf. Intell. Energy Power Syst. (IEPS), 1-5 (2014)

[14] Hutson, Chris, Ganesh Kumar Venayagamoorthy, and Keith A. Corzine. "Intelligent scheduling of hybrid and electric vehicle storage capacity in a parking lot for profit maximization in grid power transactions. " In 2008 IEEE Energy 2030 Conference, 1-8 (2008)

[15] D. Linden and T. B. Reddy, Handbook of Batteries, 3rd ed. New York, McGraw-Hill, (2001)

[16] Qian, Kejun, Chengke Zhou, Malcolm Allan, and Yue Yuan. "Modeling of load demand due to EV battery charging in distribution systems." IEEE transactions on power systems 26, 802-810 (2010)

[17] López, Karol Lina, Christian Gagné, and Marc-André Gardner. "Demand-side management using deep learning for smart charging of electric vehicles." IEEE Transactions on Smart Grid 10, 2683-2691 (2018)

[18] Madrid, Christopher, Juan Argueta, and Jordan Smith. "Performance characterization-1999 Nissan Altra-EV with lithium-ion battery." Southern California EDISON (1999).

[19] System Advisory Model (SAM) [On-line] Website: https://sam.nrel.gov/ (2018) 